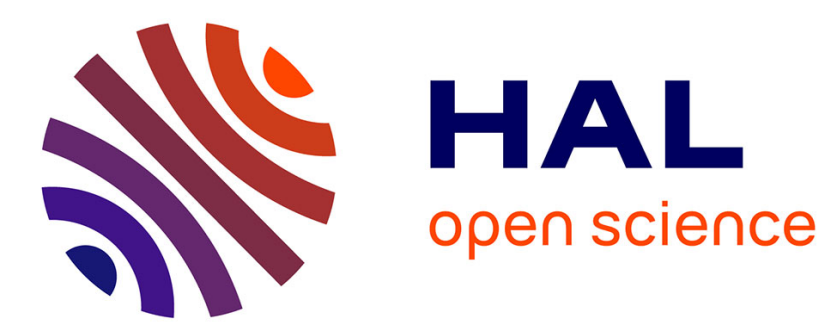

\title{
Derivation of viscous correction terms for the isothermal quantum Euler model.
}

\author{
Stéphane Brull, Florian Méhats
}

\section{To cite this version:}

Stéphane Brull, Florian Méhats. Derivation of viscous correction terms for the isothermal quantum Euler model.. Journal of Applied Mathematics and Mechanics / Zeitschrift für Angewandte Mathematik und Mechanik, 2010, 90 (3), pp.219-230. hal-01016708

\section{HAL Id: hal-01016708 https://hal.science/hal-01016708}

Submitted on 1 Jul 2014

HAL is a multi-disciplinary open access archive for the deposit and dissemination of scientific research documents, whether they are published or not. The documents may come from teaching and research institutions in France or abroad, or from public or private research centers.
L'archive ouverte pluridisciplinaire HAL, est destinée au dépôt et à la diffusion de documents scientifiques de niveau recherche, publiés ou non, émanant des établissements d'enseignement et de recherche français ou étrangers, des laboratoires publics ou privés. 


\title{
Derivation of viscous correction terms for the isothermal quantum Euler model
}

\author{
S. Brull *† $\quad$ F. Méhats ${ }^{\S \uparrow}$
}

\begin{abstract}
The aim of this paper is to compute viscous correction terms for the isothermal quantum Euler system of Degond, Gallego, Méhats (SIAM Multiscale Model Simul., 6, 2007). We derive this model by using a Chapman-Enskog expansion up to order 1. In a last part, we consider a situation where the flow is nearly irrotational in order to get a simplified model.
\end{abstract}

\section{Introduction.}

This paper is the continuation of a program of work initiated in 2003 in [8]. Extending Levermore's moments method [11] to a quantum context, P. Degond and C. Ringhofer derived hydrodynamical models from ChapmanEnskog expansions around quantum local equilibriums. Next, after this work, an entropic quantum drift-diffusion model was derived in [7], numerically discretized in [9] and discussed in the context of quantum transport in electronic nanostructures in [3]. In [5], an isothermal quantum Euler system was derived, recovering at a semiclassical asymptotics a model obtained in [10]. Extended models were also discussed in $[2,4]$.

All these models were obtained -formally- by applying a hydrodynamic or a diffusive scaling to a Wigner equation with ad-hoc relaxation operators and performing the limit as the mean free path converges to zero. In this

\footnotetext{
*Institut de Mathématiques de Toulouse

Université Paul Sabatier 118 route de Narbonne F-31062 Toulouse Cedex 9

${ }^{\dagger}$ brull@mip.ups-tlse.fr

${ }^{\S}$ IRMAR (UMR CNRS 6625), Université de Rennes, Campus de Beaulieu, 35042 Rennes Cedex (France)

Iflorian.mehats@univ-rennes1.fr
} 
paper, we are interested in the next order approximation of the hydrodynamic limit. In the classical setting, it is well-known [1] that this leads to the viscous correction to the Euler equations, i.e. to the Navier-Stokes system. Here, we apply the same idea in the quantum setting in order to derive the first order correction to the isothermal Euler equation of [5]. By analogy, we shall call quantum Navier-Stokes system the obtained model.

Let us shortly present the approach. From the Wigner equation, integrations with respect to the momentum variable $p \in \mathbb{R}^{3}$ lead to equations for the first two moments $n(t, x)$ (the mass density) and $n(t, x) u(t, x)$ (the current density), both densities being functions of the space variable $x \in \mathbb{R}^{3}$ and the time variable $t \in \mathbb{R}$ :

$$
\begin{aligned}
& \partial_{t} n+\operatorname{div}(n u)=0, \\
& \partial_{t}(n u)+\operatorname{div}(n u \otimes u+\mathbb{P})+n \nabla V=0,
\end{aligned}
$$

where $V$ denotes an applied potential. Of course, this system of equations is not closed, since the pressure tensor $\mathbb{P}$ is still expressed in terms of the microscopic Wigner function $f(t, x, p)$ :

$$
\mathbb{P}=\int_{\mathbb{R}^{d}}(p-u)(p-u) f(t, x, p) \frac{d p}{(2 \pi \hbar)^{3}},
$$

where $\hbar$ is the dimensionless Planck constant. The Wigner function $f(t, x, p)$ can be seen as a quantum extension of the phase-space Boltzmann distribution function, though $f(t, x, p)$ is not a positive function.

In [5], an isothermal quantum Euler system was derived by closing the system of moments as follows. The expression (3) of $\mathbb{P}$ is simply replaced by another one in terms of $n$ and $n u$ :

$$
\mathbb{P}=\int_{\mathbb{R}^{d}}(p-u)(p-u) f_{n, n u}^{e q} \frac{d p}{(2 \pi \hbar)^{3}},
$$

where $f_{n, n u}^{e q}$ is the so-called local equilibrium, depending only on $n$ and $n u$ in a non local (and non explicit) way - see Definition 1. Equivalently, this isothermal quantum Euler model can be obtained as the limit $\varepsilon \rightarrow 0$ of the following quantum BGK equation in a hydrodynamic scaling:

$$
\partial_{t} f+p \cdot \nabla_{x} f+\Theta(V) f=\frac{1}{\varepsilon}\left(f_{n, n u}^{e q}-f\right) .
$$

In the present paper, in order to obtain a Navier-Stokes model, $f$ will be formally expanded up to first order around the local equilibrium $f_{n, n u}^{e q}$ as $f=f_{n, n u}^{e q}+\varepsilon f_{1}$. 
The outline of the paper is the following. In Section 2, the quantum Navier-Stokes model is derived and formulated in Theorem 1. Then, Section 3 is devoted to some simplifications of this model when the flow is assumed to be nearly irrotational: Proposition 1 is a reformulation of the quantum Navier-Stokes model in this situation.

\section{Derivation of the model}

In this section we first recall some backgrounds about the Wigner equation and we present the model derived in the general situation.

\subsection{Notations and background.}

By a density operator, we shall always mean a positive, Hermitian, traceclass operator acting on $L^{2}\left(\mathbb{R}^{3}\right)$. Let us recall the definition of the first moments of a density operator $\varrho$, i.e. the mass density $n$ and the current density $n u$. These quantities are defined by duality, considering scalar test functions $\phi$ and vector ones $\Phi$. We set

$$
\begin{aligned}
\forall \phi \in C_{0}^{\infty}\left(\mathbb{R}^{3}\right) & \int n \phi d x=\operatorname{Tr}\{\varrho \phi\}, \\
\forall \Phi \in C_{0}^{\infty}\left(\mathbb{R}^{3}\right)^{3} \int n u \cdot \Phi d x= & \operatorname{Tr}\left\{\varrho W^{-1}[p \cdot \Phi]\right\} \\
= & -i \hbar \operatorname{Tr}\left\{\varrho\left(\Phi \cdot \nabla+\frac{1}{2}(\nabla \cdot \Phi)\right)\right\}
\end{aligned}
$$

Note that an immediate consequence of (5) and (6) is the following property which will be useful later:

$$
\forall \Phi-\frac{i}{\hbar} \operatorname{Tr}\{\varrho(\Phi \cdot \nabla)\}=\int n u \cdot \Phi d x+\frac{i \hbar}{2} \int n \nabla \cdot \Phi d x .
$$

In (6), $W^{-1}$ denote the inverse Wigner transform (or Weyl quantization). Let us recall the definitions of the Wigner transform and the inverse Wigner transform. The Wigner transform maps operators on $L^{2}\left(\mathbb{R}^{3}\right)$ onto symbols, ie $L^{2}\left(\mathbb{R}^{3} \times \mathbb{R}^{3}\right)$ functions of the classical position and momentum variables $(x, p) \in \mathbb{R}^{3} \times \mathbb{R}^{3}$. More precisely, one defines the integral kernel of the operator $\varrho$ to be the distribution $\varrho\left(x, x^{\prime}\right)$ such that $\varrho$ operates on any function $\psi(x) \in L^{2}\left(\mathbb{R}^{3}\right)$ as follows:

$$
\varrho \psi(x)=\int \varrho \underline{\varrho}\left(x, x^{\prime}\right) \psi\left(x^{\prime}\right) d x^{\prime} .
$$


Then, the Wigner transform $W[\varrho](x, p)$ is defined by:

$$
W[\varrho](x, p)=\int \varrho\left(x-\frac{1}{2} \eta, x+\frac{1}{2} \eta\right) e^{\frac{i \eta \cdot p}{\hbar}} d \eta .
$$

The Wigner transform can be inverted and its inverse (called Weyl quantization) is defined for any function $f(x, p)$ as the operator acting on $\psi(x) \in$ $L^{2}\left(\mathbb{R}^{3}\right)$ as:

$$
W^{-1}[f] \psi(x)=(2 \pi \hbar)^{-3} \int f\left(\frac{x+y}{2}, p\right) \psi(y) e^{\frac{i p \cdot(x-y)}{\hbar}} d p d y .
$$

We have also the following Parseval property: for any operators $\rho$ and $\sigma$ in $\mathcal{L}^{2}=\left\{\varrho: \operatorname{Tr}\left(\varrho \varrho^{\dagger}\right)<\infty\right\}:$

$$
\operatorname{Tr}\left(\rho \sigma^{\dagger}\right)=\int W[\rho] \overline{W[\sigma]} \frac{d x d p}{(2 \pi \hbar)^{3}},
$$

where the bar means complex conjugation. We also recall the cyclicity of the trace, where $a, b, c$ are three operators:

$$
\operatorname{Tr}\{[a, b] c\}=\operatorname{Tr}\{[c, a] b\}=\operatorname{Tr}\{[b, c] a\} .
$$

In quantum statistical mechanics, the Von Neumann entropy is defined by:

$$
S(\varrho)=\operatorname{Tr}(\varrho \ln \varrho-\varrho) .
$$

In order to deal with isothermal problems, we introduce the quantum free energy defined by:

$$
G(\varrho)=T S(\varrho)+E(\varrho)=\operatorname{Tr}(T(\varrho \ln \varrho-\varrho)+\mathcal{H} \varrho) .
$$

where $\mathcal{H}$ is the Hamiltonian defined by

$$
\mathcal{H}=-\frac{\hbar^{2}}{2} \Delta+V
$$

For simplicity, the potential $V=V(t, x)$ applied to the system of particles is assumed to be given, independent of the particles.

In this paper, we suppose that the particle system interacts with a thermal bath and that the mass $n$ and the current $n u$ are conserved by this interaction. Following [8], [7], [5], we shall model this interaction via a relaxation operator constructed according to a principle of free energy minimization. To this aim, we define the local thermal equilibrium associated to $n$ and $n u$, at temperature $T$ through the following formal procedure. 
Definition 1. Let the scalar function $n(x) \geq 0$ and the vectorial function $n u(x)$ be given. Consider the following constrained minimization problem:

$$
\min \{G(\varrho) \text { such that } \varrho \text { is a density operator satisfying }(5) \text { and }(6)\} \text {. }
$$

The solution, if it exists, is called the local equilibrium density operator associated to $n$ and nu. Lagrange multiplier theory for the constrained problem (14) (see [8], [5]) shows that there exist a scalar function $A$ and a vector function $B$, both real valued and defined on $\mathbb{R}^{3}$, such that this local equilibrium density operator takes necessarily the form:

$$
\varrho_{n, n u}^{e q}=\exp \left(-\frac{1}{T} H(A, B)\right),
$$

where $H(A, B)$ is the following modified Hamiltonian:

$$
H(A, B)=W^{-1}\left[\frac{1}{2}(p-B)^{2}+A\right]=\frac{1}{2}(i \hbar \nabla+B)^{2}+A .
$$

The local equilibrium Wigner function associated to $n$ and $n u$ is the Wigner transform of $\varrho_{n, n u}^{e q}$ :

$$
f_{n, n u}^{e q}=W\left[\varrho_{n, n u}^{e q}\right]
$$

\subsection{Derivation of the quantum Navier-Stokes system}

Let us now introduce the quantum BGK equation, by adding a relaxation operator to the quantum Liouville equation. This operator, denoted by $\mathcal{Q}(\varrho)$, describes the collisions between the particles and a surrounding environnement at temperature $T$. In this paper, we choose this collision operator equal to the simplest quantum BGK operator that can be constructed according to the minimization problem (14):

$$
\imath \hbar \partial_{t} \varrho=[\mathcal{H}, \varrho]+\imath \hbar \frac{\varrho_{n, n u}^{e q}-\varrho}{\varepsilon},
$$

where, for all $t$, the local equilibrium $\varrho_{n, n u}^{e q}(t)$ is defined thanks to Definition 1 from the density $n(t, \cdot)$ and the current $(n u)(t, \cdot)$ associated to $\varrho(t)$ through (5), (6).

Let us now expand $\varrho$ around $\varrho_{n, n u}^{e q}$ as follows:

$$
\varrho=\varrho_{n, n u}^{e q}+\varepsilon \varrho_{1} .
$$


By inserting this expansion of $\varrho$ into the equation (17), we get the relation defining $\varrho_{1}$

$$
\varrho_{1}=-\partial_{t} \varrho_{n, n u}^{e q}+\frac{\imath}{\hbar}\left[\mathcal{H}, \varrho_{n, n u}^{e q}\right] .
$$

By using a Wigner transformation of the equation (17), we get the following Wigner-BGK equation

$$
\partial_{t} f+p \cdot \nabla_{x} f-\Theta(V) f=\frac{f_{n, n u}^{e q}-f}{\varepsilon}
$$

with

$$
\Theta(V) f=\frac{\imath}{(2 \pi)^{3}} \int_{\mathbb{R}^{6}} \frac{V\left(t, x+\frac{\hbar}{2} \eta\right)-V\left(t, x-\frac{\hbar}{2} \eta\right)}{\hbar} f\left(x, p^{\prime}\right) e^{\imath\left(p-p^{\prime}\right) \cdot \eta} d \eta d p^{\prime} .
$$

The Wigner transformation of the expansion of $\varrho$ gives $f=f_{n, n u}^{e q}+\varepsilon f_{1}$. Hence $f_{1}$ has the expression

$$
f_{1}=-\partial_{t} f-p \cdot \nabla_{x} f+\Theta(V) f
$$

and, formally,

$$
f_{1}=-\partial_{t} f_{n, n u}^{e q}-p \cdot \nabla_{x} f_{n, n u}^{e q}+\Theta(V) f_{n, n u}^{e q}+\mathcal{O}(\varepsilon) .
$$

Moreover, by considering the first moments of (18) with respect to $p$, we obtain

$$
\partial_{t} \int f \frac{d p}{(2 \pi \hbar)^{3}}+\operatorname{div} \int p f \frac{d p}{(2 \pi \hbar)^{3}}=0
$$

and

$$
\partial_{t} \int f p \frac{d p}{(2 \pi \hbar)^{3}}+\operatorname{div} \int p \otimes p f \frac{d p}{(2 \pi \hbar)^{3}}+n \nabla_{x} V=0 .
$$

The last equation can be rewritten

$$
\partial_{t}(n u)+\operatorname{div} \int p \otimes p f_{n, n u}^{e q} \frac{d p}{(2 \pi \hbar)^{3}}+n \nabla_{x} V=-\varepsilon \operatorname{div} \int p \otimes p f_{1} \frac{d p}{(2 \pi \hbar)^{3}} .
$$

Then (20) gives formally

$$
\begin{aligned}
\partial_{t}(n u) & +\operatorname{div} \int p \otimes p f_{n, n u}^{e q} \frac{d p}{(2 \pi \hbar)^{3}}+n \nabla_{x} V \\
= & \varepsilon \operatorname{div} \int p \otimes p\left(\partial_{t} f_{n, n u}^{e q}+p \cdot \nabla_{x} f_{n, n u}^{e q}-\Theta(V) f_{n, n u}^{e q}\right) \frac{d p}{(2 \pi \hbar)^{3}}+\mathcal{O}\left(\varepsilon^{2}\right) .
\end{aligned}
$$


The isothermal quantum Navier-Stokes system is simply the model obtained when the $\mathcal{O}\left(\varepsilon^{2}\right)$ term is dropped in this equation:

$$
\begin{aligned}
& \partial_{t} n+\operatorname{div}(n u)=0, \\
& \partial_{t}(n u)+\operatorname{div} \int p \otimes p f_{n, n u}^{e q} \frac{d p}{(2 \pi \hbar)^{3}}+n \nabla_{x} V \\
& \quad=\varepsilon \operatorname{div} \int p \otimes p\left(\partial_{t} f_{n, n u}^{e q}+p \cdot \nabla_{x} f_{n, n u}^{e q}-\Theta(V) f_{n, n u}^{e q}\right) \frac{d p}{(2 \pi \hbar)^{3}},
\end{aligned}
$$

where $f_{n, n u}^{e q}$ is still defined from $n$ and $n u$ through Definition 1 .

Let us now simplify this system. Equation (22) contains moments of order 2 and 3 of the equilibrium function $f_{n, n u}^{e q}$ which might not be easy to handle. Denote

$$
\Pi_{i j}=\int p_{i} p_{j} f_{n, n u}^{e q} \frac{d p}{(2 \pi \hbar)^{3}}, \quad \mathbb{Q}_{i j k}=\int p_{i} p_{j} p_{k} f_{n, n u}^{e q} \frac{d p}{(2 \pi \hbar)^{3}} .
$$

Using commutator properties as in [5] and [4], one can express div $\Pi$ in terms of $n, n u, A$ and $B$ only. We recall from [5] that:

$$
(\operatorname{div} \Pi)_{i}=\sum_{j} \partial_{j} \Pi_{i j}=\sum_{j} \partial_{x_{j}}\left(n u_{i} B_{j}\right)+\sum_{j} n\left(u_{j}-B_{j}\right) \partial_{x_{i}} B_{j}-n \partial_{x_{i}} A .
$$

Similarly, we shall express $\sum_{j, k} \mathbb{Q}_{i j k}$ in terms of $n, n u, A, B$ and $\Pi$ only. This is the aim of the following theorem:

Theorem 1. The above defined isothermal quantum Navier-Stokes system (21), (22) is formally equivalent to the following system, up to terms of order $\mathcal{O}\left(\varepsilon^{2}\right)$ :

$$
\begin{aligned}
& \partial_{t} n+\operatorname{div}(n u)=0 \\
& \partial_{t}\left(n u_{i}\right)+\sum_{j=1}^{3}\left(\partial_{x_{j}}\left(n u_{i} B_{j}\right)+n\left(u_{j}-B_{j}\right) \partial_{x_{i}} B_{j}\right)+n \partial_{x_{i}}(V-A)=\varepsilon S_{i}
\end{aligned}
$$

where $A$ and $B$ are related to $n$ and nu via Definition 1 and where $S_{i}$ is 
equal to:

$$
\begin{aligned}
S_{i}= & -\sum_{k=1}^{3} \sum_{j=1}^{3} \partial_{x_{k}}\left(\partial_{x_{j}}\left(n u_{i} B_{j}\right) B_{k}-n \partial_{x_{j}}\left(u_{j}-B_{j}\right) \partial_{x_{i}} B_{j} B_{k}\right) \\
& -\sum_{k=1}^{3} \sum_{j=1}^{3} \partial_{x_{i}} B_{k}\left(\partial_{x_{j}}\left(n u_{k} B_{j}\right)+n \partial_{x_{j}}\left(u_{j}-B_{j}\right) \partial_{x_{k}} B_{j}\right. \\
& -\sum_{k=1}^{3} \partial_{x_{k}}\left(n \partial_{x_{i}}(V-A) B_{k}\right)+\sum_{k=1}^{3}\left(\partial_{x_{k}}\left(n u_{i} \partial_{t} B_{k}\right)+\partial_{x_{i}} \partial_{t} B_{k} n u_{k}\right) \\
& -\sum_{k=1}^{3} n \partial_{x_{i}} B_{k} \partial_{x_{k}}(V-A)+\sum_{k=1}^{3} n u_{k} \partial_{x_{i}}\left(A+\frac{B^{2}}{2}\right)-n \partial_{x_{i}} \partial_{t}\left(A+\frac{B^{2}}{2}\right) \\
& -\sum_{j=1}^{3} \sum_{j=1}^{3} \partial_{x_{j}}\left(\partial_{x_{j}}\left(A+\frac{B^{2}}{2}\right) n u_{i}+\partial_{x_{i}}\left(A+\frac{B^{2}}{2}\right) n u_{j}\right) \\
& +\sum_{j=1}^{3} \sum_{k=1}^{3} \partial_{x_{j}}\left(\partial_{x_{k}}\left(B_{k} \Pi_{i j}\right)+\partial_{x_{j}} B_{k} \Pi_{i k}+\partial_{x_{i}} B_{k} \Pi_{j k}\right) \\
& -\frac{\hbar^{2}}{4} \sum_{j=1}^{3} \sum_{k=1}^{3} \partial_{x_{j}} \partial_{x_{k}}\left(n \partial_{x_{i}} \partial_{x_{j}} B_{k}\right)+\sum_{j=1}^{3} \partial_{x_{j}}\left(\partial_{x_{j}} V n u_{i}+\partial_{x_{i}} V n u_{j}\right), \quad(27)
\end{aligned}
$$

the tensor $\Pi$ being defined by (23).

Proof. From (24), we deduce directly that the l.h.s. of (22) can be rewritten as the l.h.s. of (26). To prove the theorem, it remains to consider the r.h.s. of (22), which is

$$
-\varepsilon \operatorname{div} \int p \otimes p f_{1} \frac{d p}{(2 \pi \hbar)^{3}}=\varepsilon g_{1}+\varepsilon g_{2}+\varepsilon g_{3},
$$

where we have set, for $i=1,2,3$,

$$
\begin{aligned}
\left(g_{1}\right)_{i} & =\sum_{j=1}^{3} \partial_{t} \partial_{x_{j}} \int p_{i} p_{j} f_{n, n u}^{e q} \frac{d p}{(2 \pi \hbar)^{3}} \\
\left(g_{2}\right)_{i} & =\sum_{j=1}^{3} \sum_{k=1}^{3} \partial_{x_{j}} \partial_{x_{k}} \int p_{i} p_{j} p_{k} f_{n, n u}^{e q} \frac{d p}{(2 \pi \hbar)^{3}} \\
\left(g_{3}\right)_{i} & =-\sum_{j=1}^{3} \partial_{x_{j}} \int p_{i} p_{j} \Theta(V) f_{n, n u}^{e q} \frac{d p}{(2 \pi \hbar)^{3}} .
\end{aligned}
$$


The expressions of $g_{1}, g_{2}$ and $g_{3}$ are given in the following lemmas whose proof is in the appendix. By summing up these expressions, the proof of the theorem is complete.

Lemma 1. The quantity $g_{3}$ defined by (30) can be written

$$
\left(g_{3}\right)_{i}=\sum_{j=1}^{3} \partial_{x_{j}}\left(\partial_{x_{j}} V n u_{i}+\partial_{x_{i}} V n u_{j}\right) .
$$

Lemma 2. The quantity $g_{2}$ defined in (29) has the following expression:

$$
\begin{aligned}
\left(g_{2}\right)_{i}= & -\sum_{j=1}^{3} \sum_{j=1}^{3} \partial_{x_{j}}\left(\partial_{x_{j}}\left(A+\frac{B^{2}}{2}\right) n u_{i}+\partial_{x_{i}}\left(A+\frac{B^{2}}{2}\right) n u_{j}\right) \\
& +\sum_{j=1}^{3} \sum_{k=1}^{3} \partial_{x_{j}}\left(\partial_{x_{k}}\left(B_{k} \Pi_{i j}\right)+\partial_{x_{j}} B_{k} \Pi_{i k}+\partial_{x_{i}} B_{k} \Pi_{j k}\right) \\
& -\frac{\hbar^{2}}{4} \sum_{j=1}^{3} \sum_{k=1}^{3} \partial_{x_{j}} \partial_{x_{k}}\left(n \partial_{x_{i}} \partial_{x_{j}} B_{k}\right) .
\end{aligned}
$$

Lemma 3. Up to $\mathcal{O}(\varepsilon)$ terms, the quantity $g_{1}$ defined in (28) has the following expression:

$$
\begin{aligned}
\left(g_{1}\right)_{i}= & -\sum_{k=1}^{3} \sum_{j=1}^{3} \partial_{x_{k}}\left(\partial_{x_{j}}\left(n u_{i} B_{j}\right) B_{k}-n \partial_{x_{j}}\left(u_{j}-B_{j}\right) \partial_{x_{i}} B_{j} B_{k}\right) \\
& -\sum_{k=1}^{3} \sum_{j=1}^{3} \partial_{x_{i}} B_{k}\left(\partial_{x_{j}}\left(n u_{k} B_{j}\right)+n \partial_{x_{j}}\left(u_{j}-B_{j}\right) \partial_{x_{k}} B_{j}\right. \\
& -\sum_{k=1}^{3} \partial_{x_{k}}\left(n \partial_{x_{i}}(V-A) B_{k}\right)+\sum_{k=1}^{3}\left(\partial_{x_{k}}\left(n u_{i} \partial_{t} B_{k}\right)+\partial_{x_{i}} \partial_{t} B_{k} n u_{k}\right) \\
& -\sum_{k=1}^{3} n \partial_{x_{i}} B_{k} \partial_{x_{k}}(V-A)+\sum_{k=1}^{3} n u_{k} \partial_{x_{i}}\left(A+\frac{B^{2}}{2}\right) \\
& -n \partial_{x_{i}} \partial_{t}\left(A+\frac{B^{2}}{2}\right) .
\end{aligned}
$$

\section{The system in the irrotational case}

In this part we focus on the special case of irrotational flows, which enables to simply notably the system obtained in Theorem 1 . Indeed, it has been 
shown in [5], [4] that when $\nabla \times u=0$ then the quantity $B$ defined in Definition 1 is nothing but the velocity $u$ itself.

In the case of the isothermal quantum Euler equation, i.e. when we make $\varepsilon=0$ in (26), it has been shown in [5] that when the initial data is irrotational, then the solution remains irrotational for all time. Unfortunately, it is not clear whether this property remains true for the quantum Navier-Stokes equations (25), (26). Instead, we have the following property: if $(\nabla \times u)(t=0)=\mathcal{O}(\varepsilon)$ then we have $(\nabla \times u)(t)=\mathcal{O}(\varepsilon)$, which implies $B=u+\mathcal{O}(\varepsilon)$. In such situation, the model obtained by replacing $B$ by $u$ in the r.h.s. of (26) will only differ from $(26)$ by $\mathcal{O}\left(\varepsilon^{2}\right)$ terms, and thus will remain consistent with our theory.

Proposition 1. Assume that $(\nabla \times u)(t=0)=\mathcal{O}(\varepsilon)$. Then, for all time, we have $(\nabla \times u)(t)=\mathcal{O}(\varepsilon)$ and the following system is formally equivalent to (25), (26), up to $\mathcal{O}\left(\varepsilon^{2}\right)$ terms:

$$
\begin{aligned}
& \partial_{t} n+\operatorname{div}(n u)=0 \\
& \partial_{t}\left(n u_{i}\right)+\sum_{j=1}^{3}\left(\partial_{x_{j}}\left(n u_{i} B_{j}\right)+n\left(u_{j}-B_{j}\right) \partial_{x_{i}} B_{j}\right)+n \partial_{x_{i}}(V-A)=\varepsilon \widetilde{S}_{i}
\end{aligned}
$$

where

$$
\begin{aligned}
\widetilde{S}_{i} & =\sum_{j=1}^{3} \sum_{k=1}^{3}\left(\partial_{x_{j}}\left(\partial_{x_{j}} u_{k} \mathbb{P}_{i j}\right)+\partial_{x_{j}}\left(\partial_{x_{i}} u_{k} \mathbb{P}_{j k}\right)+\partial_{x_{j} x_{k}}^{2}\left(\mathbb{P}_{i j} u_{k}\right)\right) \\
& +\sum_{k=1}^{3} \partial_{x_{k}}\left(n u_{k}\right) \partial_{x_{i}} A-n \partial_{x_{i}}\left(\partial_{t} A\right)-\frac{\hbar^{2}}{4} \sum_{k=1}^{3} \sum_{j=1}^{3} \partial_{x_{j}} \partial_{x_{k}}\left(n \partial_{x_{i}} \partial_{x_{j}} u_{k}\right)
\end{aligned}
$$

and where $\mathbb{P}$ is the following tensor:

$$
\mathbb{P}=\int_{\mathbb{R}^{d}}(p-u)(p-u) f_{n, n u}^{e q} \frac{d p}{(2 \pi \hbar)^{d}} .
$$

Proof. If $B=u+\mathcal{O}(\varepsilon)$, then (25), (26) yield

$$
\partial_{t} u_{i}+\sum_{k=1}^{3} u_{k} \partial_{x_{k}} u_{i}+\partial_{x_{i}}(V-A)=\mathcal{O}(\varepsilon) \text {. }
$$

So $\partial_{t} \frac{u^{2}}{2}$ reads

$$
\partial_{t} \frac{u^{2}}{2}=-\sum_{j=1}^{3} \sum_{k=1}^{3}\left(u_{j} u_{k} \partial_{x_{k}} u_{j}\right)-\sum_{j=1}^{3} u_{j} \partial_{x_{j}}(V-A)+\mathcal{O}(\varepsilon) .
$$


Let us replace $B$ by $u$ in the expression (27) of $S_{i}$. The terms depending on $V$ vanish:

$$
\begin{array}{r}
-\partial_{x_{k}}\left(n u_{k} \partial_{x_{i}} V\right)-\partial_{x_{k}}\left(n u_{i} \partial_{x_{k}} V\right)+\partial_{x_{i}}\left(-\partial_{x_{k}} V\right) n u_{k}-n \partial_{x_{k}} V \partial_{x_{i}} u_{k} \\
+n \partial_{x_{i}}\left(u_{k} \partial_{x_{k}} V\right)+\partial_{x_{k}}\left(\partial_{x_{k}} V n u_{i}\right)+\partial_{x_{k}}\left(\partial_{x_{i}} V n u_{k}\right)=0 .
\end{array}
$$

Let $X$ be defined by

$$
\begin{aligned}
X & =-\partial_{x_{k}}\left(\partial_{x_{j}}\left(n u_{j} u_{i}\right) u_{k}\right)-\partial_{x_{k}}\left(n u_{i} u_{j} \partial_{x_{j}} u_{k}\right)-\partial_{x_{i}}\left(u_{j} \partial_{x_{j}} u_{k}\right) n u_{k} \\
& -\partial_{x_{i}} u_{k} \partial_{x_{j}}\left(n u_{k} u_{j}\right)+\partial_{x_{j}}\left(n u_{j}\right) u_{k} \partial_{x_{i}} u_{k}+n \partial_{x_{i}}\left(u_{k} \partial_{x_{j}} u_{k} u_{j}\right) \\
& +\partial_{x_{j}}\left(u_{k} \partial_{x_{j}} u_{k} n u_{i}\right)+\partial_{x_{j}}\left(u_{k} \partial_{x_{i}} u_{k} n u_{j}\right) .
\end{aligned}
$$

and consider the terms depending on the stress tensor $\Pi$ :

$$
\begin{aligned}
Y & =\partial_{x_{j}}\left(\partial_{x_{j}} u_{k} n u_{i} u_{k}\right)+\partial_{x_{j}}\left(\partial_{x_{i}} u_{k} n u_{j} u_{k}\right)+\partial_{x_{j} x_{k}}^{2}\left(n u_{i} u_{j} u_{k}\right) \\
& +\partial_{x_{j}}\left(\partial_{x_{j}} u_{k} \mathbb{P}_{i k}\right)+\partial_{x_{j}}\left(\partial_{x_{i}} u_{k} \mathbb{P}_{j k}\right)+\partial_{x_{j} x_{k}}^{2}\left(\mathbb{P}_{i j} u_{k}\right) .
\end{aligned}
$$

Straightforward calculations lead to

$$
X+Y=\partial_{x_{j}}\left(\partial_{x_{j}} u_{k} \mathbb{P}_{i k}\right)+\partial_{x_{j}}\left(\partial_{x_{i}} u_{k} \mathbb{P}_{j k}\right)+\partial_{x_{j} x_{k}}^{2}\left(\mathbb{P}_{i j} u_{k}\right) .
$$

Furthermore, the terms depending on $A$ become

$$
\begin{gathered}
\partial_{x_{k}}\left(n \partial_{x_{i}} A u_{k}\right)+\partial_{x_{k}}\left(n u_{i} \partial_{x_{i}} A\right)+\partial_{x_{i}}\left(\partial_{x_{k}} A\right) n u_{k} \\
+n \partial_{x_{k}} A \partial_{x_{i}} u_{k}+\partial_{x_{k}}\left(n u_{k}\right) \partial_{x_{i}} A-n \partial_{x_{i}}\left(u_{k} \partial_{x_{k}} A\right) \\
-\partial_{x_{k}}\left(\partial_{x_{k}} A n u_{i}\right)-\partial_{x_{k}}\left(\partial_{x_{i}} A n u_{k}\right)-n \partial_{x_{i}}\left(\partial_{t} A\right) \\
=\partial_{x_{k}}\left(n u_{k}\right) \partial_{x_{i}} A-n \partial_{x_{i}}\left(\partial_{t} A\right) .
\end{gathered}
$$

Hence (36) follows.

Remark 1. The semi-classical limit of (36) leads to the classical isothermal Navier-Stokes equation. Indeed, in that case, $\mathbb{P}_{i j}, A$ and $B$ have the expressions [5]

$$
\mathbb{P}_{i j}=n T \delta_{i j}, \quad A=-T \ln (n), \quad B=u .
$$

So we obtain

$$
\sum_{k=1}^{3} \sum_{j=1}^{3} \partial_{x_{j}}\left(\partial_{x_{j}} u_{k} \mathbb{P}_{i k}+\partial_{x_{i}} u_{k} \mathbb{P}_{j k}\right)=\sum_{j=1}^{3} \partial_{x_{j}}\left(\left(\partial_{x_{j}} u_{i}+\partial_{x_{i}} u_{j}\right) n T\right),
$$




$$
\begin{gathered}
-n \partial_{x_{i}}\left(\partial_{t} A\right)=-T \partial_{x_{i}}(\operatorname{div}(n u))+\frac{T}{n} \partial_{x_{i}} n \operatorname{div}(n u), \\
\operatorname{div}(n u) \partial_{x_{i}} A=-\operatorname{div}(n u) \frac{T}{n} \partial_{x_{i}} n, \\
\sum_{j=1}^{3} \partial_{x_{j}}\left(\operatorname{div}\left(u \mathbb{P}_{i j}\right)\right)=T \partial_{x_{i}} \operatorname{div}(n u),
\end{gathered}
$$

and we get finally

$$
\widetilde{S}_{i}=\sum_{j=1}^{3} \partial_{x_{j}}\left(\left(\partial_{x_{j}} u_{i}+\partial_{x_{i}} u_{j}\right) n T\right) .
$$

\section{Appendix.}

Proof of Lemma 30.

Let $f$ be given. Then we have

$$
\int p_{i} p_{j} \Theta(V) f \frac{d p}{(2 \pi \hbar)^{3}}=-\left(\partial_{\eta_{i}} \partial_{\eta_{j}} \widehat{\Theta(V) f}\right)(t, x, 0) .
$$

But from (19), it holds that

$$
\widehat{\Theta(V) f}=\imath \frac{V\left(t, x+\frac{\hbar}{2} \eta\right)-V\left(t, x-\frac{\hbar}{2} \eta\right)}{\hbar} \hat{f}(t, x, \eta) .
$$

Hence

$$
\begin{aligned}
\int p_{i} p_{j} \Theta(V) f d p=-\imath \partial_{x_{i}}\left(\frac{1}{2} \partial_{x_{j}} V\left(t, x+\frac{\hbar}{2} \eta\right)+\frac{1}{2} \partial_{x_{j}} V\left(t, x-\frac{\hbar}{2} \eta\right) \hat{f}\right. & \\
+ & \left.\frac{V\left(t, x+\frac{\hbar}{2} \eta\right)-V\left(t, x-\frac{\hbar}{2} \eta\right)}{\hbar} \partial_{\eta_{j}} \hat{f}\right)\left.\right|_{\eta=0}
\end{aligned}
$$

So

$$
\int p_{i} p_{j} \Theta(V) f \frac{d p}{(2 \pi \hbar)^{3}}=-\imath\left(\partial_{x_{j}} V \partial_{\eta_{i}} \hat{f}+\partial_{x_{i}} V \partial_{\eta_{j}} \hat{f}\right)(t, x, 0) .
$$

Then

$$
\int p_{i} p_{j} \Theta(V) f \frac{d p}{(2 \pi \hbar)^{3}}=-\partial_{x_{j}} V n u_{i}-\partial_{x_{i}} V n u_{j}
$$


where

$$
n=\int f \frac{d p}{(2 \pi \hbar)^{3}}, \quad n u=\int f p \frac{d p}{(2 \pi \hbar)^{3}},
$$

and (31) follows.

Proof of Lemma 2.

Recall the definition $(23)$ of $\mathbb{Q}$. We claim that, for all $i$ and $j$,

$$
\begin{aligned}
\sum_{k=1}^{3} \partial_{x_{k}} \mathbb{Q}_{i j k}= & -\partial_{x_{i}}\left(A+\frac{B^{2}}{2}\right) n u_{j}-\partial_{x_{j}}\left(A+\frac{B^{2}}{2}\right) n u_{i} \\
& +\sum_{k=1}^{3}\left(\partial_{x_{i}} B_{k} \Pi_{j k}+\partial_{x_{j}} B_{k} \Pi_{i k}+\partial_{x_{k}}\left(B_{k} \Pi_{i j}\right)\right) \\
& -\frac{\hbar^{2}}{4} \sum_{k=1}^{3} \partial_{x_{k}}\left(n \partial_{x_{i}} \partial_{x_{j}} B_{k}\right) .
\end{aligned}
$$

From (39), we deduce (32) and the proof of Lemma 2 is complete.

Let us prove (39). To this aim, we recall a useful commutator lemma that was established in [4]. Let us first introduce some notations. If $\alpha=$ $\left(\alpha_{1}, \alpha_{2}, \alpha_{3}\right) \in \mathbb{N}^{3}$ is a multi-index (with $\mathbb{N}$ the set of natural integers), we denote

$$
\begin{gathered}
p^{\alpha}=p_{1}^{\alpha_{1}} p_{2}^{\alpha_{2}} p_{3}^{\alpha_{3}}, \quad \partial_{x}^{\alpha}=\partial / \partial x_{1}^{\alpha_{1}} \partial / \partial x_{2}^{\alpha_{2}} \partial / \partial x_{3}^{\alpha_{3}}, \quad|\alpha|=\alpha_{1}+\alpha_{2}+\alpha_{3}, \\
\sum_{0 \leq \gamma \leq \alpha}=\sum_{\gamma_{1}=0}^{\alpha_{1}} \sum_{\gamma_{2}=0}^{\alpha_{2}} \sum_{\gamma_{3}=0}^{\alpha_{3}} \text { and } \quad\left(\begin{array}{c}
\alpha \\
\gamma
\end{array}\right)=\left(\begin{array}{c}
\alpha_{1} \\
\gamma_{1}
\end{array}\right)\left(\begin{array}{c}
\alpha_{2} \\
\gamma_{2}
\end{array}\right)\left(\begin{array}{c}
\alpha_{3} \\
\gamma_{3}
\end{array}\right),
\end{gathered}
$$

where $\left(\begin{array}{c}\alpha_{i} \\ \gamma_{i}\end{array}\right)$ are the binomial coefficients.

Lemma 4 (Lemma 3.4 of [4]). Let $\alpha=\left(\alpha_{1}, \alpha_{2}, \alpha_{3}\right) \in \mathbb{N}^{3}$ and $\beta=\left(\beta_{1}, \beta_{2}, \beta_{3}\right) \in$ $\mathbb{N}^{3}$ be two multi-indices and let $\lambda(x)$ and $\mu(x)$ be any smooth real or complex valued functions. Let us denote $\left[\lambda p^{\alpha}, \mu p^{\beta}\right]_{\hbar}$ the symbol associated to the commutator of the operators $W^{-1}\left(\lambda p^{\alpha}\right)$ and $W^{-1}\left(\mu p^{\beta}\right)$, i.e.:

$$
\left[\lambda p^{\alpha}, \mu p^{\beta}\right]_{\hbar}=W\left(\left[W^{-1}\left(\lambda p^{\alpha}\right), W^{-1}\left(\mu p^{\beta}\right)\right]\right) .
$$

The following formal expansion holds:

$$
\left[\lambda p^{\alpha}, \mu p^{\beta}\right]_{\hbar}=\sum_{k=0}^{\lfloor(|\alpha+\beta|-1) / 2\rfloor} \imath \hbar^{2 k+1}\left(-\frac{1}{4}\right)^{k}\left[\lambda p^{\alpha}, \mu p^{\beta}\right]_{2 k+1},
$$


with

$$
\left[\lambda p^{\alpha}, \mu p^{\beta}\right]_{2 k+1}=\sum_{\substack{0 \leq \gamma \leq \alpha, 0 \leq \zeta \leq \beta \\
|\gamma+\zeta|=2 k+1}}(-1)^{|\gamma|}\left(\begin{array}{c}
\alpha \\
\gamma
\end{array}\right)\left(\begin{array}{l}
\beta \\
\zeta
\end{array}\right)\left(\partial_{x}^{\zeta} \lambda\right)\left(\partial_{x}^{\gamma} \mu\right) p^{\alpha+\beta-\gamma-\zeta},
$$

where $\lfloor\cdot\rfloor$ denotes the floor function.

Let $\mu(x)$ be a given test function. From Lemma 4, we compute:

$$
\sum_{k=1}^{3} \partial_{x_{k}} \mu p_{i} p_{j} p_{k}=\frac{\imath}{\hbar}\left[\frac{|p|^{2}}{2}, \mu p_{i} p_{j}\right]_{\hbar} .
$$

Hence, an integration by parts gives

$$
\begin{aligned}
\int \mu \sum_{k=1}^{3} \partial_{x_{k}} \mathbb{Q}_{i j k} d x & =-\sum_{k=1}^{3} \int \partial_{x_{k}} \mu p_{i} p_{j} p_{k} f_{n, n u}^{e q} \frac{d x d p}{(2 \pi \hbar)^{3}} \\
& =-\frac{\imath}{\hbar} \int\left[\frac{|p|^{2}}{2}, \mu p_{i} p_{j}\right]_{\hbar} f_{n, n u}^{e q} \frac{d x d p}{(2 \pi \hbar)^{3}} \\
& =-\frac{\imath}{\hbar} \int W\left(\left[-\frac{\hbar^{2}}{2} \Delta, W^{-1}\left(\mu p_{i} p_{j}\right)\right]\right) f_{n, n u}^{e q} \frac{d x d p}{(2 \pi \hbar)^{3}} .
\end{aligned}
$$

Now, using the Parseval property (10) and observing that

$$
-\frac{\hbar^{2}}{2} \Delta=H(A, B)-W^{-1}\left(-B \cdot p+A+\frac{B^{2}}{2}\right),
$$

where $H(A, B)$ is defined by (16), we get

$$
\begin{aligned}
\int \mu & \sum_{k=1}^{3} \partial_{x_{k}} \mathbb{Q}_{i j k} d x=-\frac{\imath}{\hbar} \operatorname{Tr}\left(\left[-\frac{\hbar}{2} \Delta, W^{-1}\left(\mu p_{i} p_{j}\right)\right] \varrho_{n, n u}^{e q}\right) \\
& =-\frac{\imath}{\hbar} \operatorname{Tr}\left(\left[H(A, B)-W^{-1}\left(-B \cdot p+A+\frac{B^{2}}{2}\right), W^{-1}\left(\mu p_{i} p_{j}\right)\right] \varrho_{n, n u}^{e q}\right) \\
& =\frac{\imath}{\hbar} \operatorname{Tr}\left(\left[W^{-1}\left(-B \cdot p+A+\frac{B^{2}}{2}\right), W^{-1}\left(\mu p_{i} p_{j}\right)\right] \varrho_{n, n u}^{e q}\right) \\
& =\frac{\imath}{\hbar} \int\left[-B \cdot p+A+\frac{B^{2}}{2}, \mu p_{i} p_{j}\right]_{\hbar} f_{n, n u}^{e q} \frac{d x d p}{(2 \pi \hbar)^{3}}
\end{aligned}
$$

where we used the cyclicity of the trace and the crucial fact that, by (15), $\varrho_{n, n u}^{e q}$ commutes with the operator $H(A, B)$. 

deduce

Let us now compute $\left[-B \cdot p+A+\frac{B^{2}}{2}, \mu p_{i} p_{j}\right]_{\hbar}$. From Lemma 4, we

$$
\left[A+\frac{B^{2}}{2}, \mu p_{i} p_{j}\right]_{\hbar}=\imath \hbar\left(\partial_{x_{i}}\left(A+\frac{B^{2}}{2}\right) p_{j}+\partial_{x_{j}}\left(A+\frac{B^{2}}{2}\right) p_{i}\right) \mu,
$$

and that

$$
\left[B \cdot p, \mu p_{i} p_{j}\right]_{\hbar}=\imath \hbar\left[B \cdot p, \mu p_{i} p_{j}\right]_{1}-\frac{\imath h^{3}}{4}\left[B \cdot p, \mu p_{i} p_{j}\right]_{3},
$$

with

$$
\begin{aligned}
{\left[B \cdot p, \mu p_{i} p_{j}\right]_{1} } & =\sum_{k=1}^{3}\left[B_{k} p_{k}, \mu p_{i} p_{j}\right]_{1} \\
& =\sum_{k=1}^{3}\left(\partial_{x_{i}} B_{k} \mu p_{j} p_{k}+\partial_{x_{j}} B_{k} \mu p_{i} p_{k}-B_{k} \partial_{x_{k}} \mu p_{i} p_{j}\right)
\end{aligned}
$$

and

$$
\left[B \cdot p, \mu p_{i} p_{j}\right]_{3}=\sum_{k=1}^{3}\left[B_{k} p_{k}, \mu p_{i} p_{j}\right]_{3}=-\sum_{k=1}^{3} \partial_{x_{i}} \partial_{x_{j}} B_{k} \partial_{x_{k}} \mu .
$$

Hence,

$$
\begin{aligned}
-\left[B \cdot p, \mu p_{i} p_{j}\right]_{\hbar} & =-\imath \hbar \sum_{k=1}^{3}\left(\partial_{x_{i}} B_{k} p_{j} p_{k}+\partial_{x_{j}} B_{k} p_{i} p_{k}\right) \mu \\
& -\imath \hbar \sum_{k=1}^{3}\left(\frac{\hbar^{2}}{4} \partial_{x_{i}} \partial_{x_{j}} B_{k}-B_{k} p_{i} p_{j}\right) \partial_{x_{k}} \mu
\end{aligned}
$$


We get finally, after an integration by parts,

$$
\begin{aligned}
\int & \mu \sum_{k=1}^{3} \partial_{x_{k}} \mathbb{Q}_{i j k} d x \\
= & -\int\left(\partial_{x_{i}}\left(A+\frac{B^{2}}{2}\right) p_{j}+\partial_{x_{j}}\left(A+\frac{B^{2}}{2}\right) p_{i}\right) \mu f_{n, n u}^{e q} \frac{d x d p}{(2 \pi \hbar)^{3}} \\
& +\int \sum_{k=1}^{3}\left(\partial_{x_{i}} B_{k} p_{j} p_{k}+\partial_{x_{j}} B_{k} p_{i} p_{k}\right) \mu f_{n, n u}^{e q} \frac{d x d p}{(2 \pi \hbar)^{3}} \\
& -\int \sum_{k=1}^{3} \partial_{x_{k}}\left(\left(\frac{\hbar^{2}}{4} \partial_{x_{i}} \partial_{x_{j}} B_{k}-B_{k} p_{i} p_{j}\right) f_{n, n u}^{e q}\right) \mu \frac{d x d p}{(2 \pi \hbar)^{3}} \\
= & -\int\left(\partial_{x_{i}}\left(A+\frac{B^{2}}{2}\right) n u_{j}+\partial_{x_{j}}\left(A+\frac{B^{2}}{2}\right) n u_{i}\right) \mu d x \\
& +\int \sum_{k=1}^{3}\left(\partial_{x_{i}} B_{k} \Pi_{j k}+\partial_{x_{j}} B_{k} \Pi_{i k}\right) \mu d x \\
& -\int \sum_{k=1}^{3} \partial_{x_{k}}\left(\frac{\hbar^{2}}{4} n \partial_{x_{i}} \partial_{x_{j}} B_{k}-B_{k} \Pi_{i j}\right) \mu d x .
\end{aligned}
$$

Therefore, since this expression holds true for all function $\mu(x)$, one can identify (39).

\section{Proof of Lemma 3.}

From (24), we deduce

$$
\begin{aligned}
\left(g_{1}\right)_{i} & =\partial_{t}(\operatorname{div} \Pi)_{i} \\
& =\sum_{k=1}^{3} \partial_{x_{k}}\left(\partial_{t}\left(n u_{i}\right) B_{k}\right)+\sum_{k=1}^{3} \partial_{x_{k}}\left(n u_{i} \partial_{t} B_{k}\right)+\sum_{k=1}^{3} \partial_{x_{i}} \partial_{t} B_{k} n u_{k} \\
& +\sum_{k=1}^{3} \partial_{x_{i}} B_{k} \partial_{t}\left(n u_{k}\right)-\partial_{t} n \partial_{x_{i}}\left(A+\frac{B^{2}}{2}\right)-n \partial_{x_{i}} \partial_{t}\left(A+\frac{B^{2}}{2}\right) .
\end{aligned}
$$

But according to (21), (22), we have

$$
\begin{aligned}
\partial_{t} n & =-\operatorname{div}(n u), \\
\partial_{t}\left(n u_{i}\right) & =-\sum_{j=1}^{3} \partial_{x_{j}}\left(n u_{i} B_{j}\right)-n \sum_{j=1}^{3} \partial_{x_{j}}\left(u_{j}-B_{j}\right) \partial_{x_{i}} B_{j}-n \partial_{x_{i}}(V-A)+\mathcal{O}(\varepsilon) .
\end{aligned}
$$


So, neglecting the $\mathcal{O}(\varepsilon)$ terms, we get the following relations:

$$
\begin{aligned}
\partial_{t}\left(n u_{i}\right) B_{k} & =-\sum_{j=1}^{3} \partial_{x_{j}}\left(n u_{i} B_{j}\right) B_{k}-n \sum_{j=1}^{3} \partial_{x_{j}}\left(u_{j}-B_{j}\right) \partial_{x_{i}} B_{j} B_{k} \\
& -n \partial_{x_{i}}(V-A) B_{k}, \\
\sum_{k=1}^{3} \partial_{x_{i}} B_{k} \partial_{t}\left(n u_{k}\right) & =\sum_{k=1}^{3} \partial_{x_{i}} B_{k}\left(-\sum_{j=1}^{3} \partial_{x_{j}}\left(n u_{k} B_{j}\right)-n \sum_{j=1}^{3} \partial_{x_{j}}\left(u_{j}-B_{j}\right) \partial_{x_{k}} B_{j}\right. \\
& \left.-n \partial_{x_{k}}(V-A)\right)
\end{aligned}
$$

and

$$
\partial_{t} n \partial_{i}\left(A+\frac{1}{2} B^{2}\right)=-\sum_{k=1}^{3}\left(n u_{k}\right) \partial_{x_{i}}\left(A+\frac{1}{2} B^{2}\right)
$$

which enable to simplify (44) and gives (33).

\section{References}

[1] C. Bardos, F. Golse, D. Levermore, Fluid dynamic limits of kinetic equations. I. Formal derivations. J. Statist. Phys. 63 (1991), no. 1-2, 323-344.

[2] J.-P. Bourgade, P. Degond, F. Méhats, C. Ringhofer, On quantum extensions to classical Spherical Harmonics Expansion / Fokker-Planck models, J. Math. Phys. 47 (2006), no. 4, 043302

[3] P. Degond, S. Gallego, F. Méhats, An entropic Quantum Drift-Diffusion model for electron transport in resonant tunneling diodes, J. Comput. Phys. 221 (2007), 226-249.

[4] P. Degond, S. Gallego, F. Méhats. On Quantum Hydrodynamic and Quantum Energy Transport Models, Commun. Math.Sci. 5 (2007), no. 4, 887-908.

[5] P. Degond, S. Gallego, F. Méhats. Isothermal quantum hydrodynamics: derivation, asymptotic analysis and simulation, SIAM Multiscale Model. Simul. 6 (2007), no.1, 246-272.

[6] P. Degond, F. Méhats, C. Ringhofer, Quantum hydrodynamic models derived from the entropy principle, Contemp. Math. 371 (2005), 107331. 
[7] P. Degond, F. Méhats, C. Ringhofer, Quantum energy-transport and drift-diffusion models, J. Stat. Phys. 118 (2005), no. 3-4, 625-665.

[8] P. Degond, C. Ringhofer, Quantum Moment Hydrodynamics and the Entropy Principle, J. Stat. Phys. 112 (2003), no. 3-4, 587-628.

[9] S. Gallego, F. Méhats, Entropic discretization of a quantum driftdiffusion model, SIAM J. Numer. Anal. 43 (2005), no. 5, 1828-1849.

[10] A. Jüngel, D. Matthes, A derivation of the isothermal quantum hydrodynamic equations using entropy minimization, Z. Angew. Math. Mech. 85 (2005), 806-814.

[11] C. D. Levermore, Moment closure hierarchies for kinetic theories, J. Stat. Phys. 83 (1996), 1021-1065. 